\title{
Kindling: Secondary Epileptogenesis, Sleep and Catecholamines
}

\author{
MITSUMOTO SATO AND TOYOJI NAKASHIMA
}

SUMMARY: Seizure development and transference phenomenon were investigated in hippocampal and amygdaloid kindled cats. The behavioral and electrographic findings during the kindling procedures showed that motor seizure development in hippocampal seizures occurred with the emergence of independent discharging in the amygdala, globus pallidus and contralateral hippocampus. Furthermore, secondary site convulsions developed upon the first stimulation of these structures in the hippocampal group but only after over a month of hippocampal stimulation in the amygdaloid group. It was, therefore,

RÉSUMÉ: Une étude comparative du développement des convulsions et du phénomène de transfert après "kindling" hippocampien ou amydgalien chez le chat fut faite. Il est conclu que le rôle de l'amygdale et du globus pallidus dans le développement des convulsions hippocampiennes est plus grand que celui de concluded that role of the amygdala and globus pallidus in hippocampal seizure development was more essential than that of hippocampal stimulation in amygdaloid seizure development. The common findings between the hippocampal and amygdaloid kindled animals were the systematic progression to seizures, the all-or-nothing nature of the electrical response and the relative permanency of the seizure susceptibility. Seizure susceptibility increased during slow wave sleep and decreased during REM sleep. These latter findings were examined with preliminary data of brain bioassays of catecholamines.

l'hippocampe dans le développement des convulsions amygdaliennes. D'autres expériences montrent que la susceptibilité aux convulsions après "kindling", est nettement augmentée pendant la phase de sommeil à ondes lentes et diminuée lors de la phase sommeil-REM.
From the Department of Neuropsychiatry, School of Medicine, Okayama University.

Reprint requests to Dr. M. Sato, Department of Neuropsychiatry, School of Medicine, Okayama University Hospital, Okayama City, Japan.

\section{INTRODUCTION}

Since Goddard et al. (1969) demonstrated the progressive increase of seizure susceptibility resulting from repetitive electrical stimulation ("kindling effect"), many investigators have examined the parameters of this phenomenon: The evidence suggests that the kindling effect does not result from a localized histological change at the stimulated site, but depends upon widely disseminated neuro-circuits being constructed trans-synaptically (Goddard et al., 1969; Racine, 1972; Racine et al., 1972; Wada and Sato, 1974; Wada et al., 1974). This view is further supported by the transference phenomenon, which is the efficient "re-kindling" in a secondary brain structure after establishing the kindling effect in a primary structure. Moreover, the permanency of behavioral modification and of electrographic seizure discharge was confirmed in kindled animals (Goddard et al., 1969; Goddard, 1972; McIntyre and Molino, 1972; Wada et al., 1974). These findings strongly suggest that the kindling procedure can lead to secondary functional alterations and a predictable reorganization of brain function, culminating in a permanent state of epileptogenicity that is strikingly similar to human epilepsy.

Under the kindling effect as an experimental model of epilepsy, this report presents the results of our recent studies showing, firstly, the differences between hippocampal and amygdaloid seizures, and secondly, the correlations between kindled seizure susceptibility and various stages of the sleep-arousal continuum. 


\section{METHODS}

Twenty-three adult cats were used. Deep electrodes were implanted stereotaxically under pentobarbital anesthesia $(35 \mathrm{mg} / \mathrm{kg})$. The electrodes were made from 0.20 $\mathrm{mm}$ stainless steel wire tapered at the tip. One electrode was insulated with glass tubing to within $1 \mathrm{~mm}$ of the tip. Two adjacent electrodes were attached to the outside of the glass tubing. Bipolar recordings were obtained from the insulated electrode and one of the adjacent electrodes. The other adjacent electrode was used for electrical stimulation. Electrodes were implanted into the globus pallidus (GP), dorsal hippocampus (HIPP), lateral nucleus of amygdala (AM), putamen (PT), dorso-medial thalamic nucleus (DM), centromedial thalamic nucleus $(\mathrm{CM})$, substantia nigra $(\mathrm{SN})$, midbrain reticular formation (MRF) and the fronto-parietal cortical area. The animals for sleep study were implanted with electrodes in the lateral geniculate body and periorbicular bone to record the pontogeniculate-occipital waves (PGO waves) and eye movements.

Stimulation was administered once daily with 1 second trains of 60 $\mathrm{hz}$ sine wave at an intensity just sufficient to induce after-discharge (AD threshold). The stimulation was regulated with a Nihonkoden Constant Current Unit. The EEG was recorded for 10 minutes before and after stimulation with a polygraph. Stimulations were continued until the onset of generalized convulsive seizure, and for an additional 5 days. Subsequently, the stimulation intensity was gradually reduced each day until the generalized convulsions ceased. The minimal stimulus intensity necessary to induce seizure was designated as the "final electroconvulsive threshold".

The transference phenomenon was investigated in five hippocampal kindled cats and four amygdaloid kindled cats after the final electroconvulsive threshold was determined at the primary site. A secondary site (bilateral AM, HIPP, MRF, $\mathrm{CM}$ or contralateral HIPP) was selected and stimulated with 1 second trains of $60 \mathrm{hz}$ sine wave at an intensity of $300 \mu \mathrm{A}$. The intensity was increased in steps to 500,700 and $1000 \mu \mathrm{a}$, unless AD was triggered. After inducing epileptic manifestations, daily stimulation was delivered to the structure until a generalized convulsion was triggered.

\section{A. DIFFERENCES BETWEEN HIPPOCAMPAL AND AMYGDALOID SEIZURES}

Numerous experimental studies have been conducted on the neurophysiological basis of temporal lobe epilepsy, and the hippocampus and amygdala are believed to play important roles. However, there is little agreement on their detailed functional contributions to temporal lobe epilepsy and on the correlations between these two structures. Some comparisons will be made of the effects of hippocampal kindling (Sato, in press) and of amygdaloid kindling reported last year (Wada and Sato, 1974).

\section{Behavioral Seizure Development}

The hippocampal kindled group (HIPP group) required significantly $(p<0.01)$ more days of stimulation (mean 51.8 days) than the amygdaloid kindled group (AM group, mean 25.5 days) to develop a generalized convulsive seizure. The chronological pattern of seizure development was classified into 6 stages in both the HIPP and AM groups (Fig. 1, Table 1).

A characteristic finding of hippocampal seizure development was the presence of autonomic seizure manifestations without motor seizure manifestations in stages 1 to 3 . The period from stage 1 to $3 \mathrm{oc}-$ cupied $44.8 \%$ of the total hippocampal kindling time. However, the subsequent stages 4 to 6 involving the motor seizure manifestations showed a similar pattern to the AM group.

\section{Electrographic Findings}

Self-sustained $A D$ and interictal discharge (IID) were found entirely independent of discharges from the primary sites in both the HIPP and AM groups.

In the HIPP group, self-sustained $A D$ and/or independent IID appeared in the AM and GP in stage 4, when the animals began to respond with motor seizure manifestations,
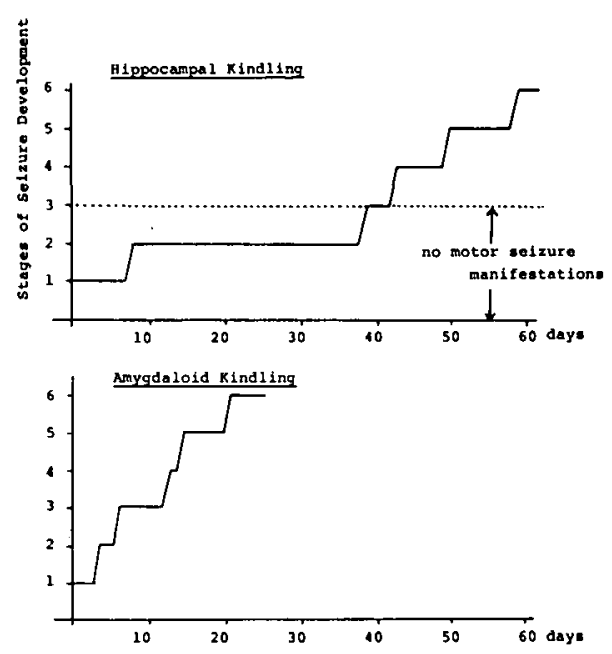

Figure 1-Number of days of daily stimulation at various stages of seizure development in representative hippocampal and amygdaloid kindled cats. Stimulation was once daily with 1 second trains of $60 \mathrm{hz}$ sine waves at an intensity just sufficient to induce after-discharge. For definitions of Stage 1-6, see Table 1.

TABLE 1

Developmental Stages of Hippocampal and Amygdaloid Seizures

\begin{tabular}{|c|l|l|}
\hline Stages & \multicolumn{1}{|c|}{ Hippocampal Kindling } & \multicolumn{1}{c|}{ Amygdaloid Kindling } \\
\hline 1 & Attention Response & Ipsilateral Facial Twitching \\
2 & Immobility & Bilateral Facial Twitching \\
3 & Autonomic Manifestations & Head Nodding \\
4 & Facial Twitching, Head & Tonic Extension of Contra- \\
& Nodding, Mastication & lateral Forepaw \\
5 & Tonic Extension of Contra- & Generalized Clonic Jerking \\
6 & lateral Forepaw & Generalized Convulsion \\
\hline
\end{tabular}


and these discharges appeared also in the MRF in stages 5 and 6.

On the other hand in the AM group, the MRF was involved in self-sustained $A D$ and independent IID in stage 3 . This secondary epileptogenesis in the MRF further disseminated into the contralateral $\mathrm{AM}$ and the bilateral GP and PT.

Another difference was present in the latency of IID. The latency between the negative peaks of bilateral hippocampal IID was within 10 msec., while inter-AM latency was $30-45 \mathrm{msec}$. regardless of direction of discharge propagation. This result was consistent with the previous finding of Buser et al (1969).

\section{Transference Phenomenon}

In the HIPP group, the transference phenomenon was confirmed in bilateral AM, GP and contralateral HIPP in all cases. Furthermore, the transference phenomenon was confirmed in MRF in one animal and in $\mathrm{CM}$ in another animal. It is particularly important to stress that in all cases of bilateral GP and contralateral HIPP the secondary site convulsions were triggered by the first stimulation at an intensity just sufficient to induce AD (Table 2).

On the other hand, from 25 to 39 (mean 32.0) hippocampal stimulations were necessary to induce secondary site convulsions in the AM group. This duration was longer than that required for AM secondary site convulsions in the HIPP group, but shorter than for HIPP primary site convulsions $(p<0.01)$.

These results taken together consistently show that the HIPP and AM may be involved in temporal lobe epilepsy in different ways. Hippocampal seizures, initially having characteristic seizure manifestations without any motor signs, develop generalized convulsions more slowly than amygdaloid seizures. Thereafter, the motor seizure development of the hippocampal seizures may depend upon secondary functional alterations of the $A M$ and GP, while the establishment of secondary epileptogenesis in HIPP is not an essential condition for amygdaloid seizure development.

\section{B. COMMON FINDINGS BETWEEN HIPPOCAMPAL AND AMYGDALOID KINDLING SEIZURES \\ 1. The Gradual Progression of Seizure Manifestations}

The animals always displayed an orderly progression through stage 1 to 5 , even when they developed generalized convulsions. In the HIPP group, mean generalization time from the end of stimulation to the animal falling down with convulsions was 42.4 (range 24.5-54.8) seconds, while the total mean duration of the final stage of convulsions was 81.7 seconds. This gradual progression indicates that the kindled convulsion is a secondary generalized convulsion. The neural events occurring during the long latency of 42.4 seconds remain unclear. This latency is independent both of structures involved in AD and of the latency for inter-hemispheric discharge propagation, since AD occurred in all recording sites by the end of stage 1 , and the latency for interamygdaloid discharge propagation was within $45 \mathrm{msec}$. According to our previous finding that desynchronization of the cortical EEG occurred in the hemisphere contralateral to the stimulated side corresponding to the long latency, it might be speculated that, besides the kindled neuro-circuits, diffuse projection systems may manipulate the seizure generalization.

\section{The "All-or-Nothing" Property}

The kindled convulsions had an all-or-nothing response to the final electroconvulsive threshold. The reduction of stimulus intensity from $A D$ threshold to the final electroconvulsive threshold was not accompanied by regression or modification of behavioral seizure manifestation and AD. Animals stimulated below the final electroconvulsive threshold did not manifest any evidence of behavioral or electrographic seizure.

Furthermore, the secondary site convulsions that were triggered by the first stimulation also had the allor-nothing property. These results suggest that kindled neuro-circuits respond as a whole to the primary or secondary site stimulation. The electroconvulsive threshold was between 100-300 $\mu$ a regardless of the variation of $\mathrm{AD}$ threshold (range 200-1000 $\mu$ a), or the locations of the primary site, reflecting the relatively constant excitability of the kindled neuro-circuits.

\section{Permanency}

The permanency of the induced seizure susceptibility is suggested by the stability of the final electroconvulsive threshold and IID, as well as the kindling effect that remained unchanged without further stimulation for more than several months. This stability is further supported by the occurrence of recurrent spontaneous seizures of focal onset in the AM group (Wada et al., 1974).

Some aspects of the kindled neuro-circuits which are reflected by these findings are: 1) the sequential firing patterns of the neuro-circuits, 2) the all-or-nothing response to primary or secondary site stimulation, 3) a stable and relatively constant seizure susceptibility and 4) the

TABLE 2

Number of Stimulations Required for Seizure Development in the Secondary Site of the Hippocampal Kindled Group

\begin{tabular}{|ccccc|ccccc|}
\hline Case No. & \multicolumn{4}{c|}{ Ipsilateral Side } & \multicolumn{5}{c|}{ Contralateral Side } \\
\hline & AM & GP & CM & MRF & HIPP & AM & GP & CM & MRF \\
1 & 1 & 1 & - & - & 1 & 3 & 1 & - & - \\
2 & 1 & 1 & - & - & 1 & 2 & 1 & - & - \\
3 & 1 & 1 & 1 & - & 1 & 1 & 1 & - & - \\
4 & 6 & 1 & - & - & 1 & 1 & 1 & - & - \\
5 & 1 & 1 & - & - & 1 & 1 & - & - & - \\
- No after-discharge was triggered. & & & & & \\
\hline
\end{tabular}


possibility that diffuse projection systems modulate the electrical activity of the neuro-circuits to induce seizure generalization.

\section{CORRELATIONS BETWEEN SEIZURE SUSCEPTIBILITY AND VARIOUS SLEEP STAGES}

The possible relationship of human epileptic seizures to the sleep-arousal levels is well documented, as Janz (1962) classified human epilepsy into awaking epilepsy, sleep epilepsy and diffuse epilepsy. This relation, however, has not been consistently confirmed in animal experiments. As an example, seizure susceptibility, reflected by interictal discharge or electroconvulsive threshold, has been reported to increase during slow wave sleep and decrease during REM sleep (Cadilhac and Passonant, 1964; Cohen et al., 1970; Frank, 1970) or to have the opposite effect (Fujimori, 1966; Hayashi, 1966; Popoviciu et al., 1971; Wilczak, 1970). The reasons for these contradictory results could be due to differences in experimental models of epilepsy, the maturation or dissemination of secondary epileptogenesis, the location of primary focus or species differences of experimental animals. We felt it would be of great interest to investigate this correlation with hippocampal kindled cats.

Nine hippocampal kindled adult cats were used. The frequency of interictal discharges and the final electroconvulsive thresholds were monitored in each of 5 sleep-arousal states: 1) an excited arousal state; 2 ) a clearly awake state in resting posture; 3) in light sleep, near sleep onset indicated by a sleeping posture accompanied by moderate amplitude slow EEG waves; 4) in moderate sleep, with high amplitude slow waves partially intermingled with PGO waves; and 5) in REM sleep, with clear evidence of REM sleeptonic EMG suppression, frequent appearance of PGO waves and an arousal pattern of cortical and hippocampal EEG. Monitoring was performed between 5 and $12 \mathrm{p} . \mathrm{m}$.

The frequency of interictal discharge in the hippocampal kindled animals was recorded in each of 3 $x_{n \rightarrow 2}$

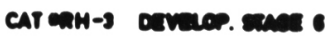

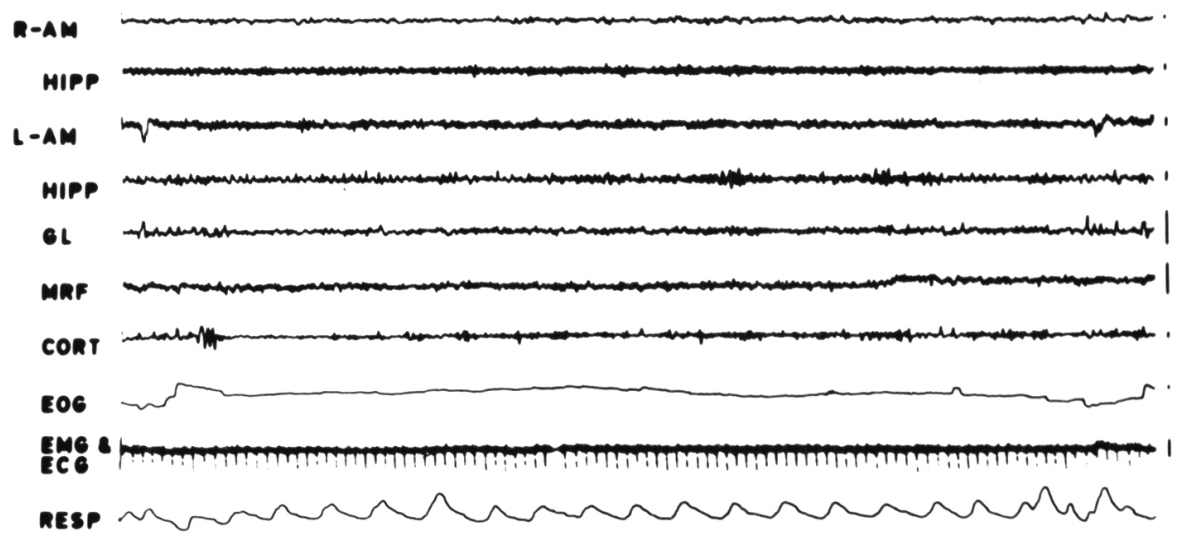

Isec.

sto

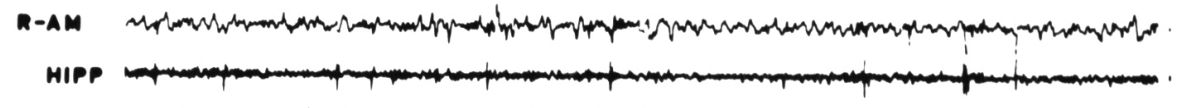

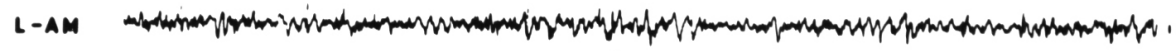

HIPP P

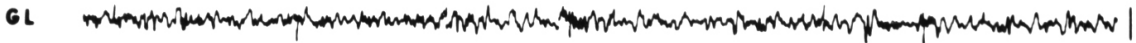

MRF

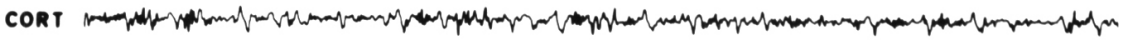

EOG

EMG

RESP
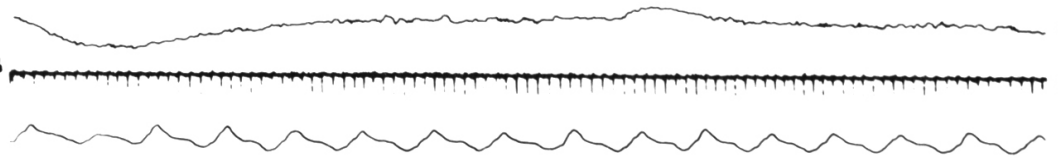

$1 \sec 100 \pi$
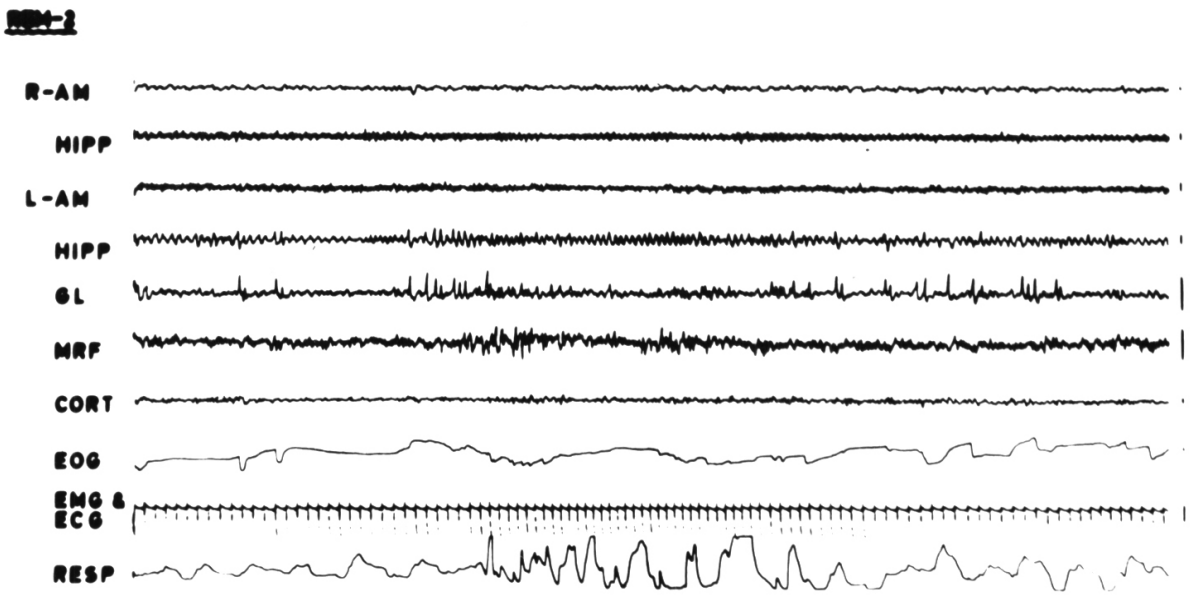

Figure 2-Frequency of interictal discharge in awake, slow wave sleep and REM sleep.

Note the marked increase of interictal discharges during slow wave sleep (SL-M) and their suppression during REM sleep (REM 2).

Abbreviations: AW-R, awake-resting; AM, amygdala; GL, lateral geniculate body; MRF, midbrain reticular formation; CORT, fronto-parietal cortex; EOG, eye movements; EMG, electromyogram; ECG, electrocardiogram; RESP, respiration. 
seizure developmental stages: 1) before stimulation; 2) during stages 3 or 4 ; and 3 ) during generalized convulsions of stage 6 .

The final electroconvulsive threshold was tested only in stage 6 . Two stimulus intensities, namely the final electroconvulsive threshold in the awake-resting state and the subthreshold intensity at $25 \mu$ a below the threshold, were delivered in each of the 3 sleep stages once every day.

The findings in these animals showed that IID increased significantly during slow wave sleep (especially moderate sleep), but disappeared during REM sleep (Fig. 2).

This correlation was constant, regardless of the seizure development stage (Fig. 3).

The lowest electroconvulsive threshold was found in slow wave sleep (especially moderate sleep) in all cases. This was evident when subthreshold stimulations were applied, although stimulation at the threshold intensity indicated no behavioral or electrographic differences in the induced seizures (Fig. 4).

These results indicate that kindled seizure susceptibility is modified along with the sleep stages, as reflected by IID frequency and electroconvulsive threshold. It was further demonstrated that this correlation of increased seizure susceptibility in slow wave sleep and definitive decreased susceptibility during REM sleep is consistent with the previous findings of Cohen et al., (1970) that the lowest threshold generally occurs during slow wave sleep with phasic activity in unkindled cats. The present study supports their view that the low threshold in slow wave sleep may be due to the summation of the applied stimuli with on-going phasic activity, and that the low voltage high frequency EEG of the REM sleep has some function in offsetting a convulsion.

Although there are many factors involved, one possible explanation for this correlation between seizure susceptibility and sleep stages is that it is closely related to biogenic amine metabolism in the brain, since many reports indicate that the serotonergic and catecholaminergic systems play an important role in slow wave sleep and in REM sleep, respectively. The role of the brain catecholaminergic systems as the neurohumoral basis of seizure susceptibility has been promoted by studies examining the effects of intraventricular injection of 6-hydroxydopamine (6-OHDA), which can produce selective damage to catecholaminergic neurons. Corcoran et al (1974) reported recently that rats with substantial depletions of both norepinephrine (NE) and dopamine (DA) following central administration of 6-OHDA required less than half as many stimulations to kindle seizures as did undepleted rats, confirming the earlier report of Arnold et al. (1973) that central catecholaminergic systems tend to inhibit kindling seizure development. These findings are consistent with the previous reports that increased seizure susceptibility occurred when brain catecholamine levels were significantly lowered, while decreased susceptibility was found when brain dopamine levels were significantly elevated (Boggan and Seiden, 1971; Bourn et al., 1972; Izumi et al., 1973; McKenzie and Soroko, 1972). Together with these findings, it is strongly suggested that the kindled seizure suspectibility may be dependent upon the depletion of brain catecholamine levels.

Preliminary bioassay data from our laboratory (Sato et al., 1975) of catecholamines in hippocampal kindled cat brains is shown in Fig 5, which indicates equal and marked depletions of both NE and DA from both hemispheres. There was no evidence of significant differing rates of depletion of NE and DA. This data suggests that kindled seizure susceptibility, which appears to be based upon widely disseminated secondary functional alterations of the brain, also has a neurohumoral basis in the depletion of catecholamine levels. According to these findings, we might speculate that seizure susceptibility, which is based on marked depletions of both

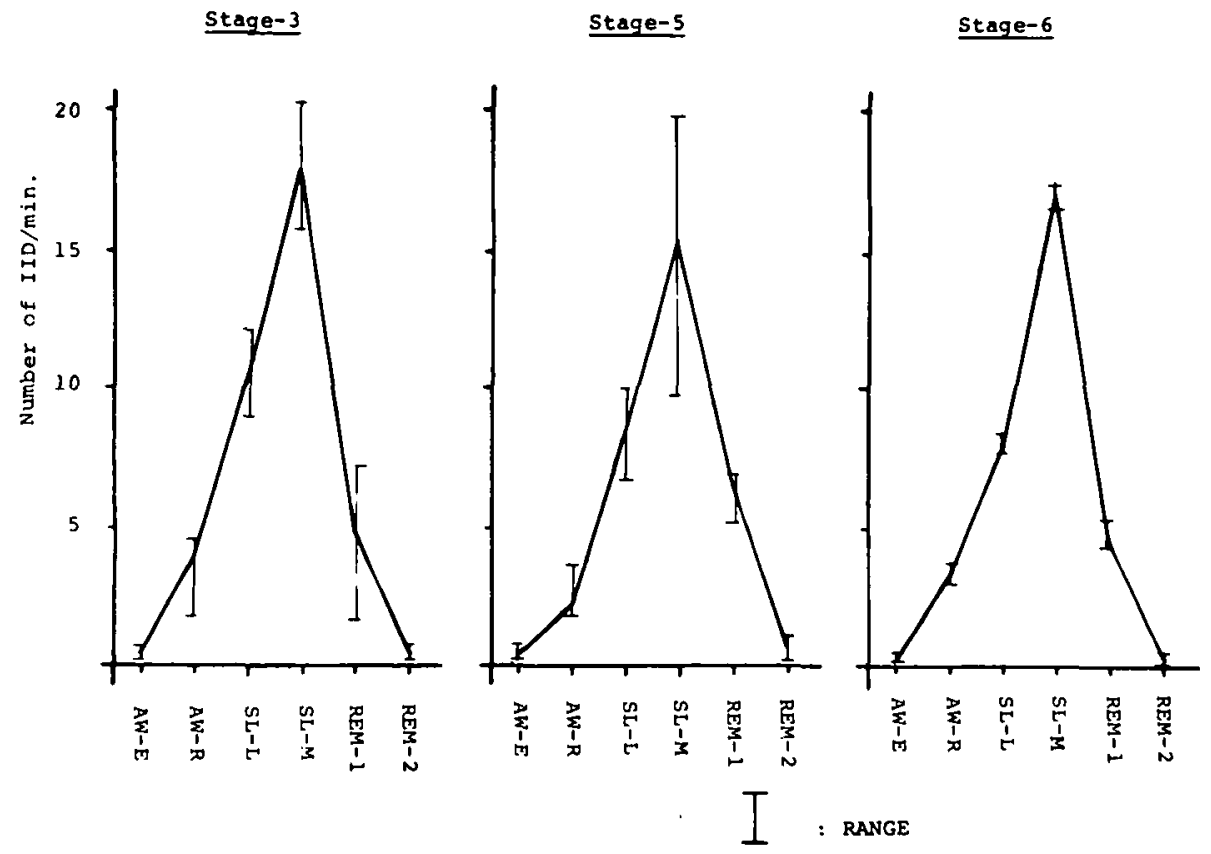

Figure 3-Mean frequency of interictal discharges in developmental stages 3, 5 and 6 in hippocampal kindled cats.

Interictal discharge increased during moderate sleep and decreased during REM sleep.

Abbreviations: AW-E, awake-exciting; AW-R, awake-resting; SL-L, light sleep; SL-M, moderate sleep; REM-1, atypical REM sleep without clear desynchronization in cortical EEG; REM-2, typical REM sleep. For definitions of Stage 3, 5 and 6, see Table 1. 


\section{AnMrE-risting}

L- $\mathbf{A M}$

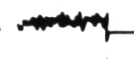

HIPP سم

GL

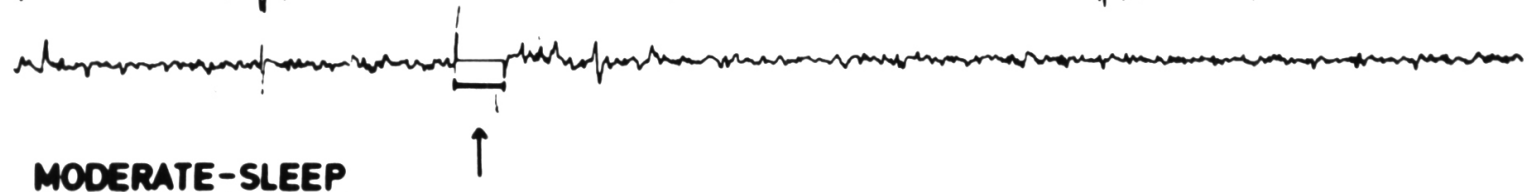

MODERATE-SLEEP

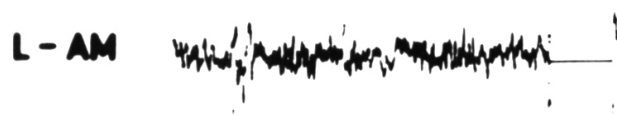

HIPP

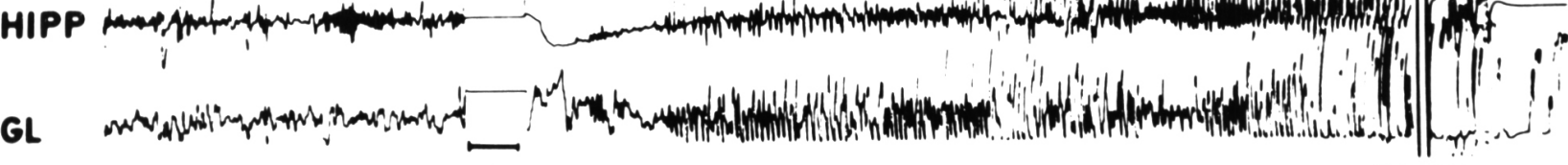

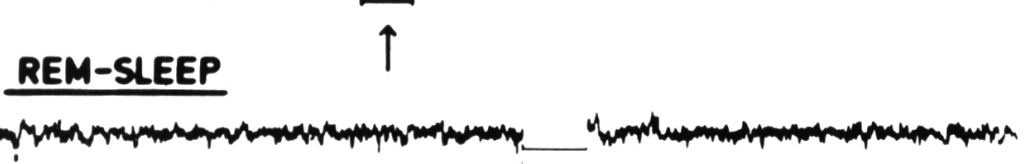

SOSEC.

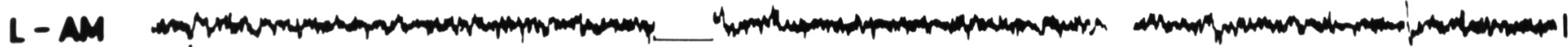

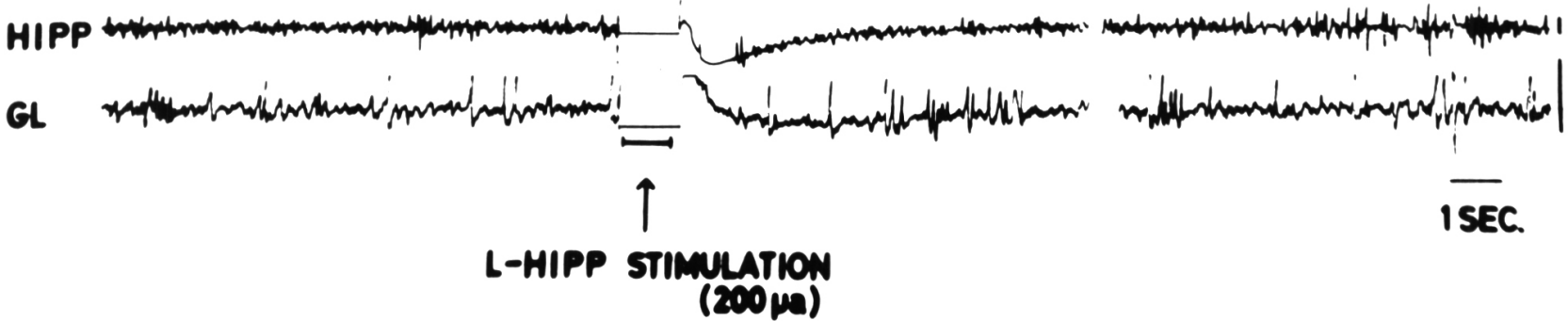

Figure 4-Subthreshold hippocampal stimulation in awake, slow wave sleep and REM sleep.

Generalized convulsions were triggered only to stimulation administered in slow wave sleep (SL-M).

NE and DA, is increased by the participation of activated serotonergic systems during slow wave sleep and is decreased by the physiologically activated catecholaminergic systems during REM sleep.
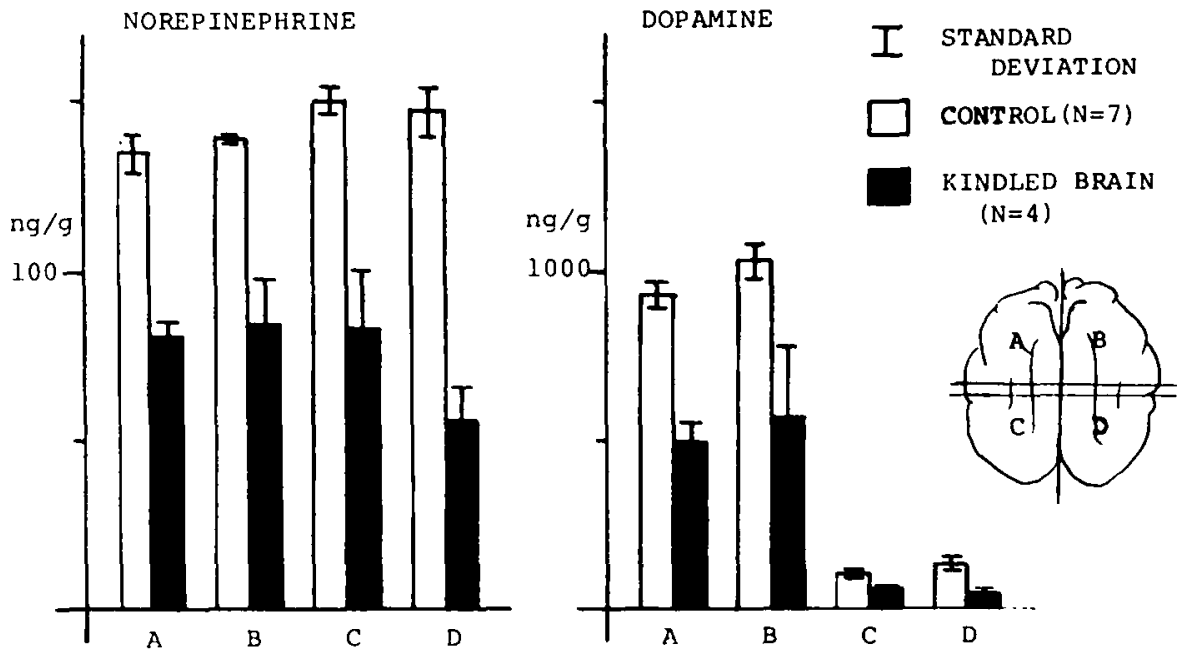

Figure 5-Norepinephrine and dopamine levels of hippocampal kindled brains. Four brain regions were sampled.

\section{DISCUSSION}

Dr. Racine: I made the point in my talk that the data we have on the kindling phenomenon do not yet provide a very compelling case in favor of mechanisms involving an increase in strength of excitatory synaptic transmission. Your data on catecholamine depletion in kindled animals might provide some support for the hypothesis that kindling involves a decrease in tonic levels of inhibition. It seems to me that that hypothesis could explain all of the data so far collected on the kindling phenomenon. Do you, or does anyone else here, have any ideas on this possibility? Dr. Morrell raised the question about the appearance of interictal spiking in rats. Actually, we see it in most of the hooded rats that we test. and it appears to be similar to the spiking reported by Dr. Morrell for the frog and $\mathrm{Dr}$. Wada for the cat, although the density is usually less than in those species. Dr. Stevens questioned the role of protein synthesis as studied by Dr. Morrell, in the kindling effect in view of her understanding that kindling will occur with very short intertrial intervals as long as afterdischarges are triggered. Dr. Goddard has demonstrated an interstimulation interval effect on kindling and we have studied it in more detail. With stimulations separated by 15 minutes, kindling will progress normally for the first 4-5 discharges. 
If separated by 30 minutes, kindling progresses normally for the first 8-9 discharges, and at one hour intervals we found no significant differences compared to animals stimulated once every 24 hours. These changes are still pretty rapid but it is possible that, like short term and long term memory or the transient and permanent phases of post-tetanic potentiation, that the changes "massed" kindling stimulations may have a different mechanism than that underlying the permanent changes that result from those stimulations. Dr. Wada: I am very pleased to see his nice result with respect to catecholamines. About four years ago, when Dr. Sato was in my laboratory, we tried to tackle this by injecting 60HDA into substantia nigra. After about six cats all of which showed inconsistent results, we abandoned it. Now he seems to have found the right track which should open up further avenues of insight into this most intriguing phenomenon. I would like to say one more thing. and that is that his finding of accentuation of interictal discharge during the slow wave stage and elimination of it during the REM stage, is exactly what we have found in both rhesus monkeys and baboons. I seem to remember, on the other hand if I am mistaken please correct me, Jan, that in your telemetry study, interictal discharge tended to increase or fluctuate greatly during the REM stage predisposing the patient to ictus? Dr. Stevens: On the contrary, Juhn, I think that our findings were just the same. That telemeter round-the-clock EEG's of man show a marked increase of spikes during slow wave sleep. a total suppression during REM, but actual frank seizures, if they occur, might occur at the beginning of the REM period or replace the REM period altogether. Your report about a low catecholamine level is consistent with Bowers and Shaywit's recent report of low HVA and HIAA in the spinal fluid of generalized epilepsy and I am wondering whether the kindling may not work through the GABA system. The nervous system ought to respond with a lot of $\mathrm{GABA}$ to kindling and that in turn would suppress the catecholamine system, if our current information about those feedback loops is correct, so that the catecholamine suppression might be secundary. Do you have any measurements of that? Dr. Sato: No, but I want to examine that possibility in the future. Dr. Penry: Dr. Susumu Sato, Dr. Dreifuss, and myself have studied long term telemetered EEG's in children with generalized spike-wave paroxysms, during wakefulness and sleep. We found that generalized spike wave paroxysms during wakefulness and less frequently during REM sleep. Generalized spike-wave paroxysms increased in Stage 2, became shorter in duration in a synchronous period. In slow wave sleep. generalized spike-wave paroxysms did not occur, but there was an increased number of nonsynchronous spikes, polyspikes, spike with slow waves which were irregular and often biphasic. Consequently, I have one question concerning your report of an increase in interictal discharge (? spontaneous hypersynchronous potentials) during slow wave sleep after kindling. Did the morphology of the interictal discharge change during slow wave sleep? Dr. Sato: I will have to examine further using areas of primary focus. We plan to kindle with cortical origins, cingulate and mesocortical areas. These interictal spikes may be influenced by the site of the primary focus. It will require further study. $D r$. Wada: Since Dr. Stevens raised the question of the possible role of GABA. I would like Dr. Corcoran to comment on our experience with 3-mercaptoproprionic acid. Dr. Corcoran: I really concur with the idea that it might be worthwhile looking at levels of GABA or measuring glutamic acid decarboxylase activity in kindled animals because our preliminary data suggest that there is something peculiar about the GABAminergic systems in rats that have been kindled. We gave an injection of a drug called 3-mercaptoproprionic acid which is a highly specific and very strong inhibitor of glutamic acid decarboxylase activity and thereby depletes GABA levels severely. We found that rats that were well kindled in the amygdala, if anything, were less sensitive to this drug than non-kindled control rats. Control rats had more spontaneous seizures of longer duration, tonic extension and more violent seizures than kindled rats did in response to this drug. So I think that there might be something funny about the GABA systems that occurs with kindling. My second comment is that I believe there is a breakdown in the catacholaminergic seizure suppressant tone with kindling and the obvious experiment to do to help consolidate that argument, I think. would be to look at the prophylactic effects, if any, of catacholamine agents and the drug we're about to start with is d-amphetamine I think that there are a number of other possibilities there. My question is with regard to when you measured the levels of catacholamine in these cats, was it immediately after seizure? Dr. Sato: It was about one week after the last operation. $D r$. Corcoran: It's not likely then that the depletion is due to feedback from a seizure? $D r$. Salo: I don't think so. Dr. FernandezGuardiola: Your results on kindling interictal spiking during sleep are in agreement with the all-night recording in epileptic children of
Tassinari, et al. (1965). This REM suppression of epileptic activity is a very interesting fact. We have shown that at the final stages of clonic activity and during the early post-ictal cortical depression there appear signs of REM sleep during several seconds (Fig. 1). We have postulated that the mechanism of seizure arrest and REM sleep could be the same. Your results of decreased levels of NE and DA in kindled brains support this point. if we consider that there is a depletion of NE and DA as a result of the excessive and continuous eliciting of inhibitory mechanisms by the process of kindling itself. Dr. Sato: Yes, we have several cats already past several months around the last seizure so I want to check it with those cats.

\section{REFERENCES}

ARNOLD, P. S., RACINE, R. J. and WISE, R. A. (1973). Effects of atropine, reserpine, 6-hydroxydopamine, and handling on seizure development in the rat. Experimental Neurology, 40, 457-470.

BOGGAN, W. O. and SEIDEN, L. S. (1971). Dopa reversal of reserpine enhancement of audiogenic seizure susceptibility in mice. Physiology and Behavior, 6, 215-217.

BOURN, W. M., CHIN, L. and PICCHIONI, A. L. (1972). Enhancement of audiogenic seizure by 6-hydroxydopamine. Journal of Pharmacy and Pharmacology, 24, 913-914.

BUSER, P., BANCAUD. J., TALAIRACH, A. L. and SZIKLA, G. (1969). Amygdalohippocampal interconnections in man. Physiological study during stereotaxic explorations. Electroencephalography and Clinical Neurophysiology, 26, 637.

CADILHAC, J. and PASSONANT, P. (1964). Influence of various phases of night sleep in the epileptic discharges in man.

A

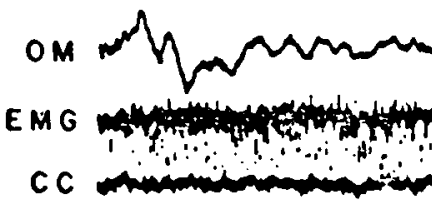

MC

c

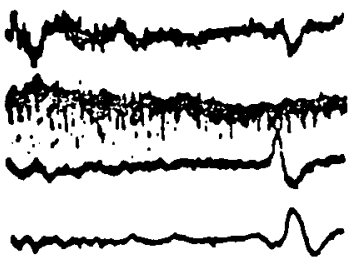

Figure I (of discussion)-Ocular movements (OM) and electromyogram of the neck muscle depression (EMG) in the last stages (extinction) of the convulsive activity. $A$, control; B, clonic phase; C, D and E, seizure arrest. CC, cerebellar cortex; $M C$, motor cortex. Note the increasing cerebellar fast activity and the appearance of rhythmic rapid ocular movements. Calibration: $100 \mathrm{msec}$. 
Electroencephalography and Clinical Neurophysiology, 17, 441.

COHEN, H., THOMAS, J. and DEMENT, W. C. (1970). Sleep stages, REM deprivation and electroconvulsive threshold in the cats. Brain Research, 19, 317-321.

CORCORAN, M. E., FIBIGER, H. C., McCAUGHRAN, J. A. and WADA, J. A. (1974). Potentiation of amygdaloid kindling and metrazol-induced seizures by 6-hydroxydopamine in rats. Experimental Neurology, 45, 118-133.

FRANK, G. (1970). Epileptiform discharge in the various stages of sleep. Electroencephalography and Clinical Neurophysiology, 28, 90-105.

FUJIMORI, F. (1966). Electroencephalographic study on the focal seizure discharges during nocturnal sleep of epileptics (in Japanese). Psychiatria et Neurologia Japonica. 68. 330-350.

GODDARD, G. V. (1972). Long term alteration following amygdaloid stimulation. In: The Neurobiology of the Amygdala, p. 581-596, Plenum Publishing Corporation, New York

GODDARD, G. V., McINTYRE, D. C. and LEECH, C. K. (1969). A permanent change in brain function resulting from daily electrical stimulation. Experimental Neurology, 25, 295.330.

HAYASHI, A. (1966). An experimental study on the relationship between the epileptic seizure discharge and natural sleep stages (in Japanese). Psychiatria et Neurologia Japonica, 68, 351-379.

IZUMI, K., DONALDSON, J., MINNICH, $\mathrm{J}$, and BARBEAU. A. (1973). Ouabain induced seizures in rats: Relation to brain monoamines. Canadian Journal of Biochemistry, 51, 198-203.
JANZ, D. (1962). The grand mal epilepsies and the sleeping-waking cycle. Epilepsia (Amst.), 3, 69-109.

McINTYRE, D. C. amd MOLINO, A. (1972). Amygdala lesions and CER learning longterm effect of kindling. Physiology and Behavior, 8, 1055-1058.

MCINTYRE, D. C. and GODDARD, G. V. (1973). Transfer, interference and spontaneous recovery of convulsions kindled from the rat amygdala. Electroencephalography and Clinical Neurophysiology, 35 . 533-543.

McKENZIE, G. M. and SOROKO, F. E. (1972). The effects of apomorphine, (十)-amphetamine and 1-dopa on maximal electroshock convulsions - a comparative study in the rat and mouse. Journal of Pharmacy and Pharmacology, 24, 696-701.

POPOVICIU, L., SZABO, L. and BORCHINA, N. (1971). Electropolygraphical all night sleep in certain form of epilepsy. Electroencephalography and Clinical Neurophysiology, 30, 361 .

RACINE, R. J. (1972). Modification of seizure activity by electrical stimulation: I. After-discharge threshold. Electroencephalography and Clinical Neurophysiology, 32, 269-279.

RACINE, R. J. (1972). Modification of seizure activity by electrical stimulation: II. Motor seizure. Electroencephalography and Clinical Neurophysiology, 32, 281-294.

RACINE, R. J., GARTNER, J. G. and MCINTYRE, B. W. (1972). Epileptiform activity and plasticity in limbic structures. Brain Research, 47, 262-268.

SATO, M. (1975). An experimental study of epilepsy with "kindling effect" procedure - I. Behavioral and electroencephalog- raphic study on the developmental process of hippocampal seizure (in Japanese). Psychiatria et Neurologia Japonica, 77, in press.

SATO, M. (1975). An experimental study of epilepsy with "kindling effect" procedure - II. Study on the secondary epileptogenesis of the hippocampal seizure with "transference phenomenon" (in Japanese). Psychiatria et Neurologia Japonica, 77, in press.

SATO, M., ONISHI, T., NAKASHIMA, T., UEFUJI, K., TAKAHASHI, Y. and OTSUKI, S. (1974). Influence of sleep-arousal levels on the hippocampal inter-ictal discharge (in Japanese). Medicine and Biology, 89, 251-256.

SATO, M. and WADA, J. A. (1975). Review on the kindling preparation: A new experimental model of epilepsy (in Japanese). Brain and Nerve, 27, 257-276.

SATO, M., NAKASHIMA, T., OTSUKI, S., KISHIKAWA, $\mathrm{H}$. and KOBAYASHI, $\mathrm{K}$. (1975). Change of brain catecholamine levels in the kindling preparation of cats (in Japanese). Igakuno Ayumi, 92, in press.

WADA, J. A. and SATO, M. (1974) Generalized convulsive seizure induced by daily electrical stimulation of the amygdala in cats: Correlative electrographic and behavioral features. Neurology, 24, 565-574.

WADA, J. A., SATO, M. and CORCORAN, M. E. (1974). Persistent seizure susceptibility and recurrent spontaneous seizures in kindled cats. Epilepsia (Amst.), 15, 465-478.

WILCZAK, H. (1970). Influence of various phases of night sleep on the epileptic discharges in man (in Polish). Psychiatria Polska, 4, 31-36. 